07

\title{
Влияние морфологии поверхности слоев InAIAs на температурные зависимости параметров диодов Шоттки Au/Ti/n-InAlAs (001)
}

\author{
() И.Б. Чистохин ${ }^{1}$, М.С. Аксенов ${ }^{1,2,}$ ศ, Н.А. Валишева ${ }^{1}$, Д.В. Дмитриев ${ }^{1}$, И.В. Марчишин ${ }^{1}$, А.И. Торопов $^{1}$, \\ К.С. Журавлев ${ }^{1,2}$ \\ ${ }^{1}$ Институт ффизики полупроводников им. А.В. Ржанова СО РАН, \\ Новосибирск, Россия \\ ${ }^{2}$ Новосибирский государственный университет, \\ Новосибирск, Россия \\ ฯ E-mail: m.se.aksenov@gmail.com
}

Поступило в Редакцию 27 ноября 2018 г.

В окончательной редакции 27 ноября 2018 г.

Принято к публикации 30 ноября 2018 г.

\begin{abstract}
Изучено влияние ростовых структурных дефектов поверхности слоев InAlAs, выращенных методом молекулярно-лучевой эпитаксии на подложках $\operatorname{InP}(001)$, на температурные зависимости вольт-амперных характеристик барьеров Шоттки Au/Ti/InAlAs. Показано, что дефекты в виде ямок являются причиной возникновения областей с пониженной высотой барьера, которые при плотности $\geq 10^{7} \mathrm{~cm}^{-2}$ оказывают существенное влияние на параметры барьера Шоттки при температурах ниже $200 \mathrm{~K}$.
\end{abstract}

DOI: 10.21883/PJTF.2019.04.47342.17609

Природа формирования барьера Шоттки (БШ) на границе раздела металл/полупроводник и связанные с ней механизмы переноса зарядов являются предметом интенсивных исследований уже несколько десятилетий $[1,2]$, поскольку свойства этой границы раздела определяют рабочие характеристики приборов, их стабильность и надежность [3,4]. Вольт-амперные характеристики (BAX) реальных БШ обычно не соответствуют идеальной модели термоэлектронной эмиссии (ТЭ). Неидеальное поведение ВАХ БШ (коэффициент идеальности больше единицы) в слаболегированном (менее $10^{16} \mathrm{~cm}^{-3}$ ) полупроводнике часто связывают с наличием естественного оксидного слоя и интерфейсных ловушек [1,5]. С другой стороны, в работе Танга [6] показано, что неидеальное поведение ВАХ может быть количественно объяснено в предположении о наличии на границе раздела металл/полупроводник локально расположенных областей с пониженной высотой барьеров, имеющих гауссово распределение, и линейными размерами порядка глубины области обеднения. Наличие статистического распределения этих областей экспериментально было подтверждено с помощью баллистической электронной микроскопии [7]. Аргументом в пользу использования модели Танга для анализа ВАХ является проявление в экспериментах аномальной корреляционной зависимости коэффициента идеальности и высоты БШ от температуры. Однако в этой модели отсутствуют предположения о природе неоднородностей в барьере Шоттки. Целью настоящей работы является установление влияния плотности ростовых структурных дефектов поверхности InAlAs на транспорт носителей заряда в диодах Шоттки Au/Ti/n-InAlAs, что является логическим продолжением предыдущей публикации [8].
Использовались гетероэпитаксиальные структуры (ГЭС) $\quad i-\operatorname{In}_{0.52} \mathrm{Al}_{0.48} \mathrm{As}(300 \mathrm{~nm}) / n^{+}-\mathrm{In}_{0.52} \mathrm{Al}_{0.48} \mathrm{As}: \mathrm{Si}$ $(500 \mathrm{~nm})$, выращенные на epi-ready полуизолирующих подложках InP (001) методом молекулярно-лучевой эпитаксии (МЛЭ) на установке „Соmpact 21T“ фирмы Riber при температуре $520^{\circ} \mathrm{C}$. При МЛЭ-росте использовались стандартная [8] $(A)$ и улучшенная $(B)$ технологии. Улучшение технологии заключалось в выращивании на поверхности InP буферного подслоя перед ростом слоев InAlAs. Состав слоев контролировался ex situ методом рентгеновской дифрактометрии. Концентрация носителей в слоях $i$-InAlAs (n-тип проводимости) и $n^{+}$-InAlAs составляла $(3-5) \cdot 10^{15}$ и $\sim 10^{18} \mathrm{~cm}^{-3}$ соответственно [8]. Изготовление барьеров Шоттки диаметром $200 \mu \mathrm{m}$ проводилось напылением слоев $\mathrm{Ti} / \mathrm{Au}(20 / 200 \mathrm{~nm})$ на поверхность InAlAs мезаструктуры после удаления остаточного оксидного слоя в растворе $\mathrm{HCl}: \mathrm{H}_{2} \mathrm{O}=1: 10$ в течение $15 \mathrm{~s}$. Меза-структуры формировались травлением ГЭС в смеси $\mathrm{H}_{3} \mathrm{PO}_{4}: \mathrm{H}_{2} \mathrm{O}_{2}: \mathrm{H}_{2} \mathrm{O}=1: 1: 38$. Омический контакт к слою $n^{+}$-InAlAs изготавливался напылением $\mathrm{Ge} / \mathrm{Au} / \mathrm{Ni} / \mathrm{Au}(20 / 40 / 20 / 200 \mathrm{~nm})$ и вжиганием контакта при $T=385^{\circ} \mathrm{C}$ в течение $5 \mathrm{~min}$ в водороде. Поверхность меза-структур пассивировалась слоем двуокиси кремния $(800 \mathrm{~nm})$. Все структуры изготавливались параллельно в одних и тех же технологических процессах. Морфология поверхности изучалась методом атомносиловой микроскопии (ACM) с помощью сканирующего зондового микроскопа Solver P-47Н. Измерения темновых ВАХ проводились в термостатированной камере c контролем температуры с помощью анализатора полупроводниковых приборов Keysight B1500A.

На рис. 1 показаны АСМ-изображения $(20 \times 20 \mu \mathrm{m})$ поверхности ГЭС $i$-InAlAs $/ n^{+}$-InAlAs/InP, выращенных 

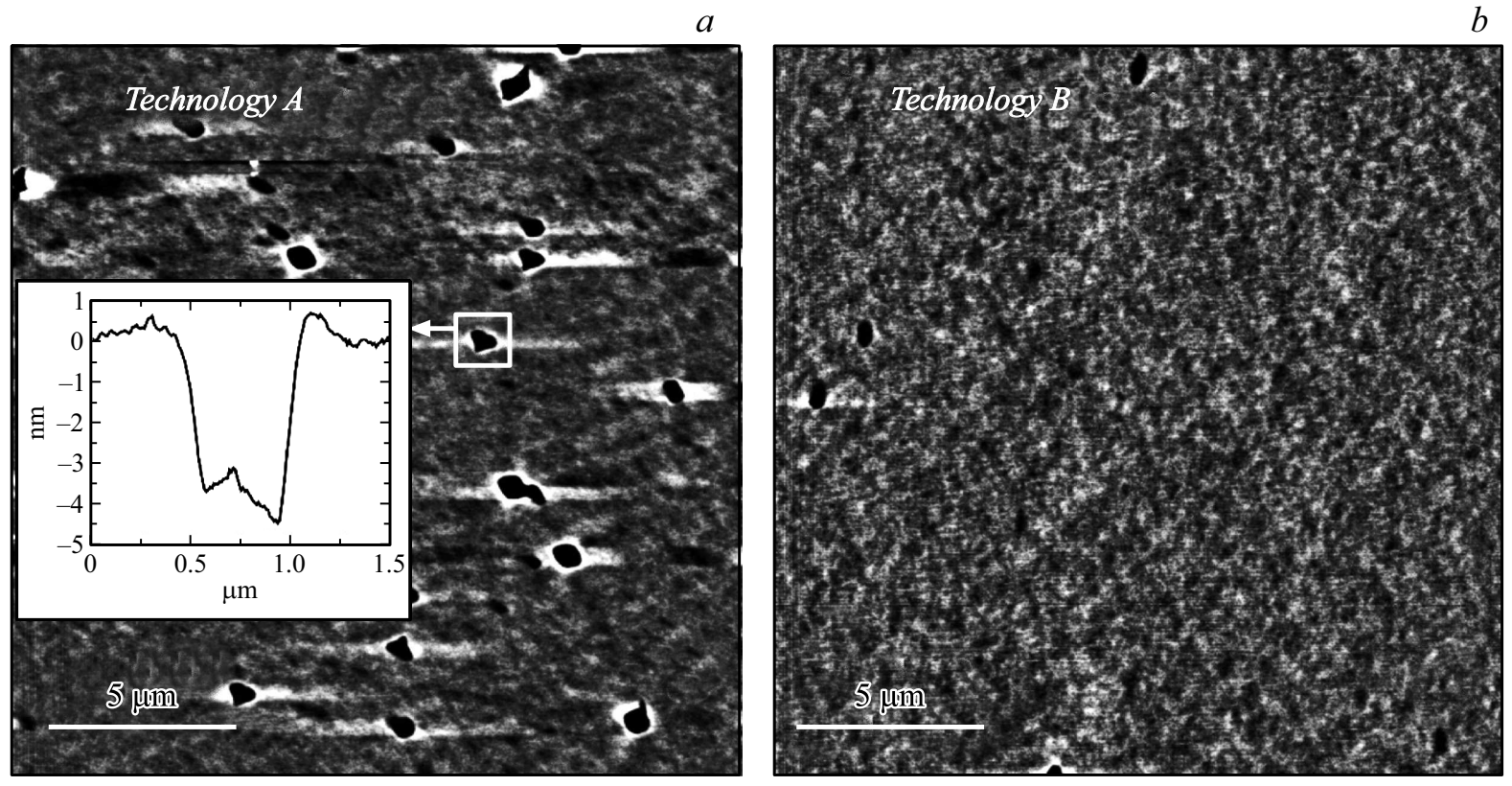

Рис. 1. АCМ-изображения $(20 \times 20 \mu \mathrm{m})$ поверхностей слоев InAlAs, выращенных методом МЛЭ по технологии $A(a)$ и технологии $B(b)$.
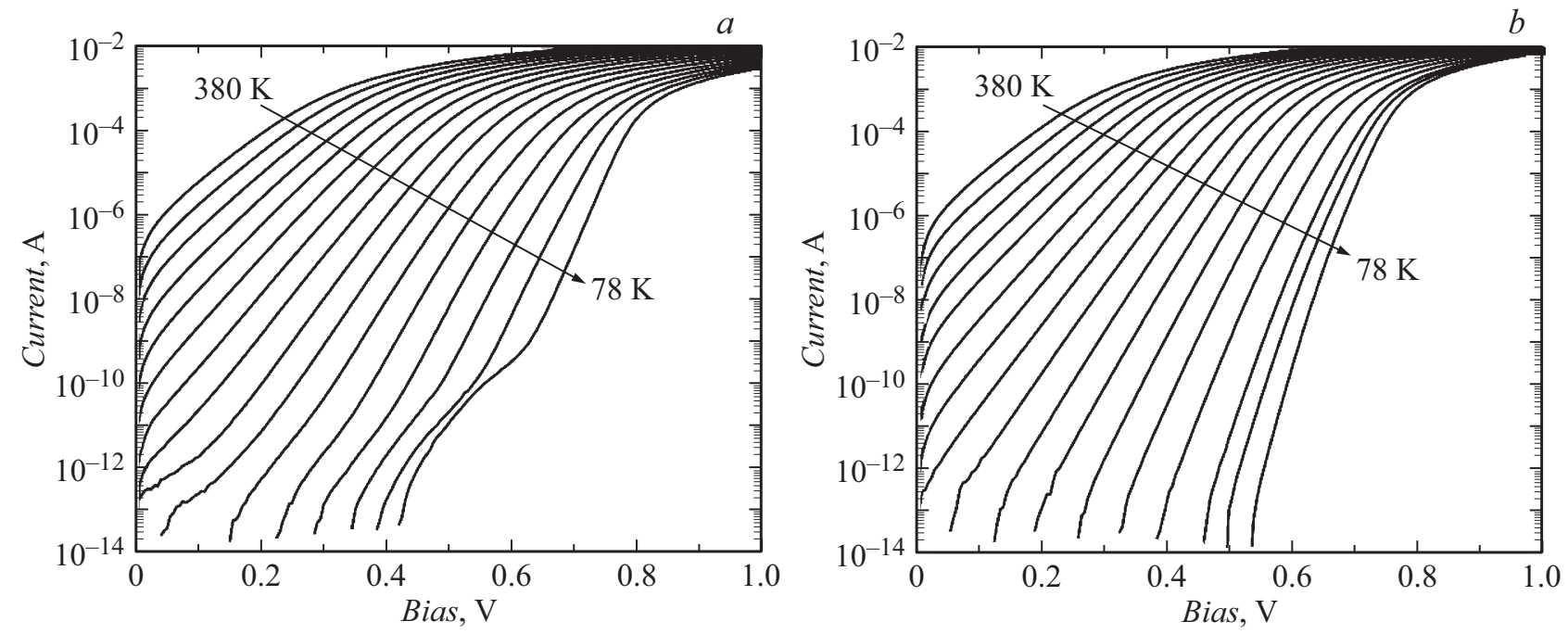

Рис. 2. Прямые ветви ВАХ диода Шоттки $\mathrm{Au} / \mathrm{Ti} / n-\mathrm{InAlAs,}$ сформированных на ГЭС, выращенных по технологии $A$ (a) и технологии $B(b)$ при температурах $78 \mathrm{~K}$, а затем при температурах от 100 до $380 \mathrm{~K}$ с шагом $20 \mathrm{~K}$.

по технологиям $A(a)$ и $B(b)$. Видно, что использование технологии роста $A$ приводит к формированию большого числа $\left(\sim 10^{7} \mathrm{~cm}^{-2}\right)$ ростовых структурных дефектов в виде ямок с глубиной от 4 до $6 \mathrm{~nm}$ и линейными размерами от 0.3 до $1 \mu \mathrm{m}$ (рис. $1, a$ ). Характерный АСМ-профиль ямочного дефекта представлен на вставке к рис. 1, $a$. Использование буферного подслоя, препятствующего прорастанию структурных дефектов с гетерограницы подложка $\mathrm{InP} /$ эпитаксиальный слой (технология $B$ ), позволяет на порядок уменьшить плотность дефектов (рис. $1, b)$. Шероховатость $(\mathrm{rms})$ на обеих поверхностях InAlAs в областях без дефектов состав- ляет $0.2-0.4 \mathrm{~nm}$. Химическая обработка поверхности InAlAs перед формированием контакта Шоттки незначительно (до $0.8-1 \mathrm{~nm}$ ) увеличивает шероховатость и не влияет на плотность ростовых дефектов.

На рис. 2 в полулогарифмическом масштабе представлены прямые ветви ВАХ БШ, изготовленных на ГЭС $i$-InAlAs $/ n^{+}$-InAlAs/InP, выращенных по технологиям $A(a)$ и $B(b)$ при температурах $78-380 \mathrm{~K}$. Из анализа прямых ветвей BAX в соответствии с теорией ТЭ [1] при использовании значения эффективной постоянной Ричардсона $A^{*}=10.1 \mathrm{~A} \cdot \mathrm{cm}^{-2} \cdot \mathrm{K}^{-2}[9]$ и геометрической площади БШ $A=3.14 \cdot 10^{-4} \mathrm{~cm}^{2}$ были 

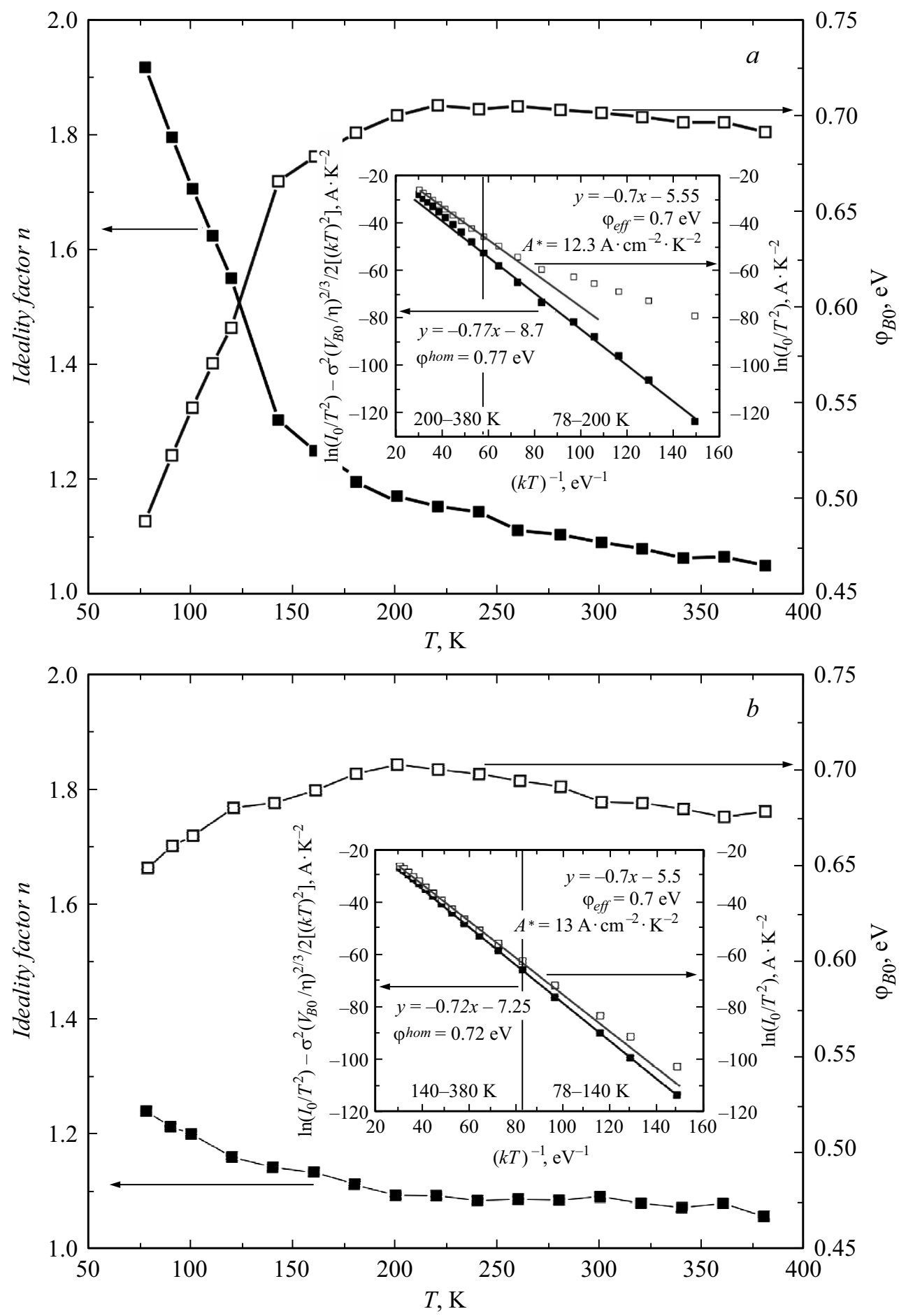

Рис. 3. Температурные зависимости $n$ и $\varphi_{B 0}$ в диапазоне $78-380$ К для БШ с высокой $(a)$ и низкой $(b)$ дефектностью. На вставках представлены графики Ричардсона БШ Аu/Ti/n-InAlAs на основе теории ТЭ (светлые символы) и модели Танга (темные символы).

определены коэффициенты идеальности $(n)$ и высоты барьеров $\left(\varphi_{B 0}\right)$ при различных температурах (рис. $\left.3, a, b\right)$. Видно, что для обоих типов гетероструктур $n$ и $\varphi_{B 0}$ в диапазоне температур 200-380 K изменяются слабо и хорошо описываются теорией ТЭ. Однако при температуре ниже $200 \mathrm{~K}$ наблюдается резкое увеличение коэффициента идеальности $n$ (от 1.1 до 1.9 ) и умень- шение высоты барьера $\varphi_{B 0}$ (от 0.7 до $0.5 \mathrm{eV}$ ) для БШ, сформированного на поверхности InAlAs с высокой плотностью $\left(10^{7} \mathrm{~cm}^{-2}\right)$ дефектов (рис. $\left.3, a\right)$. Изменение этих величин для барьера Шоттки, сформированного на поверхности InAlAs с плотностью дефектов $10^{6} \mathrm{~cm}^{-2}$, составляет для $n$ от 1.1 до 1.24 , для $\varphi_{B 0}$ от 0.7 до $0.65 \mathrm{eV}$ (рис. $3, b)$. Для обоих БШ в температурном диапазоне 
78-200 K наблюдается корреляционная линейная зависимость между $n$ и $\varphi_{B 0}$. Линейная зависимость между $n$ и $\varphi_{B 0}$ характерна для латеральной неоднородности БШ, которая может быть объяснена в рамках модели Танга [2,6], где транспорт носителей заряда носит термоактивационный характер и определяется различными высотами барьеров. Дополнительным аргументом в пользу наличия латеральной неоднородности БШ на гетероструктурах с высокой плотностью дефектов является существование участка тока с перегибом в диапазоне менее $10^{-9}$ А при малых напряжениях смещения и температурах ниже $170 \mathrm{~K}$ на экспериментальных $\mathrm{BAX}$ (рис. 2, a), что согласуется с результатами работ $[2,6]$. С использованием приближения Танга для температурных зависимостей $n$ и $\varphi_{B 0}[10]$ для обоих БШ были вычислены значения среднеквадратичного отклонения гауссова распределения высоты барьера $(\sigma)$. Оно составило $8.4 \cdot 10^{-5}$ и $3.9 \cdot 10^{-5} \mathrm{~cm}^{2 / 3} \cdot \mathrm{V}^{1 / 3}$ для БШ с плотностью дефектов на поверхности InAlAs, равной $10^{7}$ и $10^{6} \mathrm{~cm}^{-2}$ соответственно.

На вставках к рис. 3, $a, b$ для изученных барьеров Шоттки представлены график Ричардсона в координатах $\ln \left(I_{0} / T^{2}\right)-1 / k T$ на основе теории ТЭ (светлые символы) и модифицированный график Ричардсона в координатах $\ln \left(I_{0} / T^{2}\right)-\sigma^{2}\left(V_{B 0} / \eta\right)^{2 / 3} / 2(k T)^{2}-1 / k T$ на основе приближения Танга (темные символы). Здесь $I_{0}$ - ток насыщения, $T-$ температура, $k-$ постоянная Больцмана, $V_{B 0}=\varphi_{B 0}^{\text {hom }}-E_{c}-E_{F}$, где $V_{B 0}-$ изгиб зон при $V=0$ гомогенного барьера, $\varphi_{B 0}^{\text {hom }}-$ высота барьера гомогенного перехода, $\eta=\varepsilon_{s} \varepsilon_{0} / q N_{D}$, $q$ - заряд электрона, $\varepsilon_{s} \varepsilon_{0}$ - диэлектрическая постоянная полупроводника, $N_{D}-$ концентрация легирующей примеси. Из вставки к рис. 3, $a$ видно, что для БШ, сформированных на гетероструктурах InAlAs с высокой дефектностью, только в температурном диапазоне 200-380 K полученные экспериментальные данные (светлые символы) в координатах $\ln \left(I_{0} / T^{2}\right)-1 / k T$ линейны и хорошо описываются теорией ТЭ с эффективным барьером $0.7 \mathrm{eV}$. Пересечение оси ординат при $x=0$ дает значение $A^{*}=12.3 \mathrm{~A} \cdot \mathrm{cm}^{-2} \cdot \mathrm{K}^{-2}$, что достаточно хорошо согласуется с литературными данными [9]. При температуре ниже $200 \mathrm{~K}$ линейная зависимость наблюдается только в координатах модифицированного графика Ричардсона (темные символы), где наклон дает значение $\varphi_{B 0}^{h o m}=0.77 \mathrm{eV}$. Пересечение оси ординат при $x=0$ для модифицированного графика Ричардсона равно $\ln \left(N A_{e f f} A^{*}\right)$, где $N-$ количество областей с пониженной высотой барьера, а $A_{e f f}$ - эффективная площадь одной области [10]. Тогда общая площадь областей с пониженной высотой барьера $\left(N A_{e f f}\right)$, сформированных на ГЭС с высокой дефектностью, равна $1.3 \cdot 10^{-5} \mathrm{~cm}^{2}$, что составляет $\sim 4 \%$ от общей площади контакта. Для барьеров на гетероструктурах с плотностью дефектов $10^{6} \mathrm{~cm}^{-2}$ (рис. $3, b$, вставка) полученные экспериментальные данные (светлые символы) вплоть до температур $120-140 \mathrm{~K}$ линейны и хорошо описываются теорией ТЭ с эффективным барьером $0.7 \mathrm{eV}$, определяемым наклоном. Учет вклада барьеров с пониженной высотой по модели Танга (темные символы) дает высоту барьера гомогенного перехода $0.72 \mathrm{eV}$ (рис. $3, b$, вставка). Общая площадь областей с пониженной высотой барьера для данных барьеров, определенная из модифицированного графика Ричардсона, составляет $5.5 \cdot 10^{-6} \mathrm{~cm}^{2}$ или $\sim 1.8 \%$ от общей площади контакта.

Таким образом, изучение влияния морфологии поверхности слоев InAlAs, выращенных методом МЛЭ, на прямые ветви ВАХ барьеров Шоттки $\mathrm{Au} / \mathrm{Ti} / i-\mathrm{InAlAs} / n^{+}-\mathrm{InAlAs} / \mathrm{InP}$ в температурном диапазоне $78-380 \mathrm{~K}$ показало, что ростовые дефекты с плотностью $10^{6} \mathrm{~cm}^{-2}$ оказывают слабое влияние на $\mathrm{BAX}$, которые практически во всем диапазоне температур хорошо описываются теорией термоэлектронной эмиссии с одинаковым уровнем $\varphi_{B 0}$, близким к $0.7 \mathrm{eV}$. В то же время для слоев InAlAs с высокой $\left(10^{7} \mathrm{~cm}^{-2}\right)$ плотностью ямочных дефектов при температурах ниже $200 \mathrm{~K}$ наблюдаются значительные отклонения в поведении BAX от теории термоэлектронной эмиссии. Эти отклонения хорошо описываются в рамках модели Танга, предполагающей наличие на поверхности InAlAs локальных неоднородностей с пониженной высотой барьера. Полученные результаты показывают, что ростовые структурные ямочные дефекты являются причиной возникновения таких областей.

Авторы выражают благодарность А.С. Кожухову за АСМ-измерения, выполненные на оборудовании Центра коллективного пользования ИФП СО РАН „Наноструктуры“.

\section{Список литературы}

[1] Rhoderick E.H., Williams R.H. // Metal-semiconductor contacts. Oxford: Clarendon Press, 1988. 252 p.

[2] Tung R.T. // Mater. Sci. Eng. R. 2001. V. 35. P. 1-138. https://doi.org/10.1016/S0927-796X(01)00037-7

[3] Chizh A., Malyshev S., Mikitchuk K. High-speed highpower InAlAs/InGaAs/InP Schottky photodiode // 2015 Int. Topical Meeting on microwave photonics (MWP). IEEE, 2015. P. 1-4.

[4] Takahashi T., Kawano Y., Makiyama K., Shiba S., Sato M., Nakasha Y., Hara N. // IEEE Trans. Electron. Dev. 2017. V. 64. P. 89-95. DOI: 10.1109/TED.2016.2624899

[5] Omar S., Sudarshan T., Rana T., Song H., Chandrashekhar M. // J. Phys. D: Appl. Phys. 2014. V. 47. P. 295102. DOI: $10.1088 / 0022-3727 / 47 / 29 / 295102$

[6] Tung R.T. // Phys. Rev. B. 1992. V. 45. P. 13509-13523. https://doi.org/10.1103/PhysRevB.45.13509

[7] Olbrich A., Vancea J., Kreupl F., Hoffmann H. // J. Appl. Phys. 1998. V. 83. P. 358-365. https://doi.org/10.1063/1.366691

[8] Chistokhin I.B., Aksenov M.S., Valisheva N.A., Dmitriev D.V., Kovchavtsev A.P., Gutakovskii A.K., Prosvirin I.P., Zhuravlev K.S. // Mater. Sci. Semicond. Process. 2018. V. 74. P. 193-198. https://doi.org/10.1016/j.mssp.2017.10.014

[9] Hamdaoui N., Ajjel R., Salem B., Gendry M. // Mater. Sci. Semicond. Process. 2014. V. 26. P. 431-437. https://doi.org/10.1016/j.mssp.2014.05.043

[10] Korucu D., Turut A. // Int. J. Electron. 2014. V. 101. P. 1595 1606. https://doi.org/10.1080/00207217.2014.888774 